\title{
Pseudophakic astigmatism reduction with femtosecond laser-assisted corneal arcuate incisions: a pilot study
}

This article was published in the following Dove Press journal:

Clinical Ophthalmology

23 January 2017

Number of times this article has been viewed

\author{
Clayton Blehm' \\ Richard Potvin ${ }^{2}$ \\ 'Gainesville Eye Associates, \\ Gainesville, GA, ${ }^{2}$ Science in Vision, \\ Akron, NY, USA
}

Purpose: The aim of this study was to assess the effectiveness of the Verion-LenSx guided arcuate incision technique to reduce refractive astigmatism in a pseudophakic population.

Patients and methods: A prospective single-arm study was conducted in which one or both eyes of subjects required reduction of 1.0-2.0 D of refractive astigmatism after previous cataract surgery or refractive lens exchange. The surgeon used the refractive cylinder in the eye and the Woodcock astigmatism nomogram for preoperative planning, while the LenSx femtosecond laser with the Verion Image Guided System was used to create all arcuate incisions. The primary outcome measure was the uncorrected monocular distance visual acuity (UCVA). Secondary outcome measures included the change in corneal astigmatism, the change in refractive astigmatism, the best-corrected visual acuity and spectacle independence at distance from preoperative stage to 1 month and 2 months postoperatively.

Results: Twenty-eight eyes of 18 subjects were treated. The best-corrected visual acuity at the 2-month postoperative (PO) stage was not statistically significantly different from the preoperative visual acuity ( 0.02 logarithm of the minimum angle of resolution [logMAR] in both cases, $P>0.05$ ). Uncorrected visual acuity was statistically significantly better at the 2-month PO stage relative to the preoperative value $(0.14$ versus $0.34 \log \mathrm{MAR}, P<0.01)$. The mean change in refractive cylinder from the preoperative stage to the 2-month PO stage was 1.0 D. At the 2-month PO stage, two-thirds of the subjects (12/18) reported that they did not use glasses for distance vision and that their spectacle use for distance vision at 2 months was "lower" or "much lower" than the preoperative stage; in $71 \%$ of eyes $(20 / 28)$, the residual refractive cylinder was $\leq 0.50 \mathrm{D}$. Vector changes in keratometric astigmatism were weakly associated with changes in refractive cylinder.

Conclusion: Arcuate incisions made with a femtosecond laser to treat moderate levels of residual refractive astigmatism after previous cataract surgery may reduce dependence on spectacles for distance vision.

Keywords: Verion, LenSx, cylinder, cataract surgery, visual acuity, spectacle

\section{Introduction}

Significant corneal astigmatism at the time of cataract surgery is relatively common; in a large sample of older patients, corneal astigmatism was $>1.0 \mathrm{D}$ in one-third of all eyes. ${ }^{1}$ If postoperative (PO) refractive astigmatism is of approximately the same magnitude, uncorrected vision will be compromised. ${ }^{2,3}$ This can be problematic in patients who wish to reduce their dependence on spectacles, particularly for those implanted with multifocal lenses. ${ }^{4,5}$ Surgical options for reducing astigmatism after cataract extraction and lens implantation are limited to corneal refractive surgery (laser-assisted in situ keratomileusis, photorefractive keratectomy) or corneal relaxing incisions if an intraocular lens (IOL) exchange is not desired. 
Corneal relaxing incisions are one of the most common methods of reducing low-to-moderate levels of astigmatism at the time of cataract surgery. Historically, these incisions were performed manually with a knife. Results have been mixed; in a large meta-analysis, the average residual astigmatism in 225 eyes receiving relaxing incisions was as high as $1.32 \mathrm{D}$, and it was suggested that relaxing incisions may not significantly reduce spectacle dependence when compared to cases that do not receive astigmatism-correcting procedures. ${ }^{6}$ Two studies included in this meta-analysis show poor reduction of corneal astigmatism (mean change of only $0.18 \mathrm{D})^{7}$ and high spectacle dependence ( $45 \%$ in one study). ${ }^{8}$ The latter study reported that $15 \%$ of eyes had uncorrected visual acuity worse than $20 / 40$.

The introduction of femtosecond laser-assisted cataract surgery has provided surgeons with the ability to make image-guided laser arcuate incisions to correct residual astigmatism after cataract surgery. Studies show that compared to manual incisions, laser incisions likely allow for improved precision ${ }^{9}$ and may be less harmful to tissue, ${ }^{10}$ both of which may improve the predictability, safety and effectiveness of astigmatism correction. The mean difference between the intended and achieved laser incision sizes, documented to be within $0.1 \mathrm{~mm},{ }^{11}$ shows a precision that cannot be matched with manual techniques. Laser arcuate incisions have been demonstrated to significantly reduce corneal astigmatism. ${ }^{12,13}$ The incision may remain closed or may be opened if further astigmatism reduction is deemed necessary. ${ }^{12}$ Uncorrected visual acuity has been reported to improve from a mean uncorrected visual acuity of $\sim 20 / 63$ preoperatively to $\sim 20 / 30$ at 5 months postoperatively. ${ }^{14}$ Laser arcuate incisions were demonstrated in one study to provide similar effectivity as toric lenses, ${ }^{14}$ in contrast to results with manual incisions, which have been consistently demonstrated to be less effective than toric IOLs. ${ }^{6}$ Femtosecond laser systems include image guidance, allowing for great precision with regard to the location, arc length and depth of the corneal incisions they make; this is a likely source of these improved outcomes. To further improve accuracy, separate imaging systems may be used to measure corneal astigmatism and allow for proper orientation of the eye in the upright position to incisions made by the laser in the supine position.

As noted earlier, corneal arcuate incisions for the correction of astigmatism are generally performed at the time of cataract surgery. However, there is nothing to indicate that such incisions would be less effective at a later time. If created at the time of cataract surgery, incisions are generally based on anterior corneal astigmatism, which will not include the effects of surgical incisions, lens centration and posterior corneal astigmatism. Correction of astigmatism after refractive stability has been achieved can be based on the patient's manifest refraction, which will inherently include the refractive effects of the factors cited earlier. As such, it is possible that corneal arcuate incisions performed after cataract surgery may actually provide better outcomes in the correction of astigmatism.

The purpose of the current study was to assess the effectiveness of corneal arcuate incisions, planned using an image-guided system (Verion ${ }^{\circledR}$ Image Guided System; Alcon, Fort Worth, TX, USA) and created using a femtosecond laser system (LenSx ${ }^{\circledR}$; Alcon), on patients who had previous cataract surgery. The hypothesis was that the arcuate incisions would improve uncorrected distance visual acuity and reduce dependence on spectacles for distance vision.

\section{Patients and methods}

This was a prospective single-arm study of pseudophakic subjects $\geq 40$ years of age presenting for astigmatism reduction in one or both eyes; target enrollment was 30 eyes, with one or both eyes of a subject eligible for enrollment. Sample size was based on reliably detecting a one-line change in uncorrected distance visual acuity, with a $30 \%$ correction factor to adjust for likely correlation between eyes in bilaterally enrolled subjects. Subjects must have been previously implanted with a monofocal IOL, have demonstrated PO refractive stability for at least 3 months, have good ocular health with no pathology that might compromise visual acuity (outside of residual refractive error) and have 1.0-2.0 D of refractive astigmatism with a spherical equivalent refraction between $-0.50 \mathrm{D}$ and $+0.25 \mathrm{D}$. Exclusion criteria include irregular astigmatism and previous ocular surgery (besides cataract surgery). Institutional review board (IRB) approval for the study was requested and obtained (Salus IRB, Austin, TX, USA). The study was registered with clinicaltrials.gov (NCT02763124) and was conducted in compliance with the tenets of the Declaration of Helsinki and adhered to applicable regulatory requirements. Written informed consent was obtained from all study subjects.

The surgeon used the patient's refractive astigmatism data to plan the arcuate incisions. The December 2011 version of the Woodcock nomogram (Figure 1) was used to determine incision length and location. Incisions were created using the LenSx femtosecond laser system under a local anesthetic, aligning the incisions with the Verion Image Guided System (both Alcon). All eyes received paired incisions $180^{\circ}$ apart on an $8 \mathrm{~mm}$ diameter circle using $90^{\circ}$ side cuts to a depth of $90 \%$ of the cornea; pulse energy was $2 \mu \mathrm{J}$ with a spot separation of 
Woodcock LenSx astigmatism nomogram December 2011

8.0 oz $90 \%$ Depth

$6.0 \mu \mathrm{J}$ Energy

$7 \mu \mathrm{m}$ Spot separation

Age, years

\begin{tabular}{|c|c|c|c|c|c|c|c|c|c|c|c|c|}
\hline & & & & & & & & & & & & \\
\hline & & 35 & 40 & 45 & 50 & 55 & 60 & 65 & 70 & 75 & 80 & 85 \\
\hline Single & $0.50 \mathrm{D}$ & 34 & 34 & 33 & 32 & 31 & 30 & 29 & 28 & 27 & 26 & 26 \\
\hline Paired & $0.75 \mathrm{D}$ & 23 & 22 & 22 & 21 & 21 & 20 & 19 & 19 & 18 & 18 & 17 \\
\hline Paired & $1.00 \mathrm{D}$ & 34 & 34 & 33 & 32 & 31 & 30 & 29 & 28 & 27 & 26 & 26 \\
\hline Paired & $1.25 \mathrm{D}$ & 41 & 40 & 39 & 38 & 37 & 36 & 35 & 34 & 33 & 32 & 31 \\
\hline Paired & $1.50 \mathrm{D}$ & 48 & 47 & 46 & 44 & 43 & 42 & 41 & 39 & 38 & 37 & 36 \\
\hline Paired & $1.75 \mathrm{D}$ & 52 & 50 & 49 & 48 & 46 & 45 & 44 & 42 & 41 & 40 & 38 \\
\hline Paired & $2.00 \mathrm{D}$ & 55 & 53 & 52 & 51 & 49 & 48 & 47 & 45 & 44 & 42 & 41 \\
\hline Paired & $2.25 \mathrm{D}$ & 58 & 56 & 55 & 54 & 53 & 51 & 49 & 48 & 46 & 45 & 43 \\
\hline Paired & $2.50 \mathrm{D}$ & 61 & 59 & 58 & 57 & 56 & 54 & 52 & 51 & 49 & 48 & 46 \\
\hline Paired & $2.75 \mathrm{D}$ & 64 & 62 & 61 & 60 & 59 & 57 & 55 & 54 & 52 & 50 & 48 \\
\hline Paired & $3.00 \mathrm{D}$ & 67 & 65 & 64 & 63 & 62 & 60 & 58 & 56 & 55 & 53 & 51 \\
\hline
\end{tabular}

Figure I Woodcock nomogram for arcuate incision planning.

Notes: Only one arc should be used for $0.5 \mathrm{D}$ astigmatism; other amounts use paired arcs. Center on visual axis; decentered arcs may overcorrect or cause irregular astigmatism; take caution when using nomogram in red zone; consider pairing LenSx with toric IOL using the ORA ${ }^{\mathrm{TM}}$ intraoperative aberrometer (Alcon Laboratories, Inc., Fort Worth, TX, USA). If ORA is available, do not open arcs if astigmatism is not on expected axis, or if astigmatism measures <I.0 D; open later in the office PRN. Mark cornea prior to LenSx application and compensate for cyclorotation.

Abbreviations: IOL, intraocular lens; PRN, as needed; D, diopters.

$3 \mu \mathrm{m}$. Immediately following the laser treatment, the arcuate incisions were opened at an operating microscope using the surgeon's standard technique. All study subjects were placed on an appropriate antibiotic and steroid drop four times a day (QID) for 1 week, followed by only the steroid drop on a tapered dose for the following 3 weeks. All adverse events/ serious adverse events (AEs/SAEs) were recorded at the operative visit and all PO visits.

Subjects were examined at six visits: a preoperative visit, the surgical visit and follow-up visits at PO 1 day, 1 week, 1 month and 2 months. Clinical evaluations included measurement of visual acuity, manifest refraction, corneal astigmatism (with three different keratometers) and a standard slit lamp examination. All clinical evaluations were recorded at the preoperative visit and all PO visits, except for the 1-day and 1 -week visits, where only the uncorrected visual acuity was recorded. The three keratometers used were the IOLMaster 500 (Carl Zeiss Meditec, Berlin, Germany), Verion (Alcon) and autokeratometry using a handheld autorefractor (model AR-20ST1; NIDEK Co Limited, Tokyo, Japan). Preoperatively and at the 1-month and 2-month PO visits, patients were asked about their spectacle use for distance vision; the questions and possible answers are shown in Figure 2.

The primary outcome measure was the uncorrected monocular distance visual acuity (UCVA) of the eyes. Secondary outcome measures included changes in corneal astigmatism, refractive astigmatism, best-corrected visual acuity (BCVA) and spectacle independence at distance. All visual acuities were measured in Snellen notation and converted to the logarithm of the minimum angle of resolution ( $\log$ MAR) for analysis.
The data were recorded and entered in an Excel spreadsheet; this spreadsheet was imported into an MS Access database for data checking, collation and preliminary analysis (both Microsoft Corp, Redmond, WA, USA). Statistical analyses were performed using the Dell Statistica data analysis software system, version 13 (Dell, Inc, Round Rock, TX, USA). Statistical testing was performed using analysis of variance (ANOVA) on continuous variables and appropriate nonparametric tests on categorical data. Statistical significance was set at $P=0.05$. Vector math was used where appropriate for astigmatism analysis.

\section{Results}

Twenty-eight eyes of 18 subjects were treated. Demographic and preoperative data are shown in Table 1. There was a

1. How often do you use corrective lenses (glasses) for distance vision?
a. Not at all
b. Some of the time
c. Half of the time
d. Most of the time
e. All of the time

2. How would you rate your need for corrective lenses for distance vision now, compared to before the procedure?
a. Much lower
b. Lower
c. About the same
d. Higher
e. Much higher

Figure 2 Spectacle independence questionnaire.

Notes: This questionnaire was completed by subjects preoperatively (QI) and at I month and 2 months postoperatively (Q1, Q2). 
Table I Subject preoperative demographics

\begin{tabular}{|c|c|c|c|c|}
\hline Characteristics & Mean & SD & Minimum & Maximum \\
\hline Age, years & 68.8 & 6.1 & 59 & 83 \\
\hline $\begin{array}{l}\text { Days after cataract } \\
\text { surgery }\end{array}$ & 1,044 & $\mathrm{I}, 377$ & $\mathrm{I}, 0 \mathrm{I}$ & 5,517 \\
\hline $\begin{array}{l}\text { Spherical equivalent } \\
\text { refraction, D }\end{array}$ & -0.14 & 0.26 & -0.50 & 0.25 \\
\hline Refractive cylinder, D & 1.35 & 0.30 & 1.00 & 2.00 \\
\hline \multicolumn{5}{|l|}{ Average K, D } \\
\hline IOL Master & 44.27 & 1.41 & 41.62 & 46.79 \\
\hline Verion & 44.54 & 1.54 & 41.65 & 47.42 \\
\hline AutoK & 44.29 & 1.42 & 41.31 & 46.75 \\
\hline \multicolumn{5}{|l|}{$\begin{array}{l}\text { Corneal } \\
\text { astigmatism, D }\end{array}$} \\
\hline IOL Master & 1.42 & 0.54 & 0.35 & 2.26 \\
\hline Verion & 1.35 & 0.57 & 0.11 & 2.25 \\
\hline AutoK & 1.46 & 1.12 & 0.25 & 6.50 \\
\hline Arc subtense, degrees & 35.8 & 6.1 & 27.0 & 47.0 \\
\hline
\end{tabular}

Note: Total number of subjects was 18 ( 12 females, 6 males), and total number of eyes was 28 .

Abbreviations: AutoK, autokeratometry; IOL, intraocular lens; SD, standard deviation; $\mathrm{K}$, keratometry; D, diopters.

statistically significant difference in average keratometry by device $(P=0.01$ ), with the Verion device reading $\sim 0.25 \mathrm{D}$ higher than the other two devices on average. There was no statistically significant difference in the magnitude of corneal astigmatism measured by the three devices $(P=0.76)$. All preoperative corneal astigmatism measurements were $<2.4 \mathrm{D}$, with the exception of one anomalous autokeratometry reading of $6.5 \mathrm{D}$ in one eye; the average measure for the other two devices in that eye was $2.2 \mathrm{D}$.

Each patient was treated with a two-arc incision pattern of $8 \mathrm{~mm}$ diameter with $90 \%$ depth. Average arc subtense in degrees is shown in Table 1. All surgeries required between 4 seconds and 8 seconds of laser time. There were no complications at the time of surgery.

Figure 3 shows the mean UCVA and BCVA over time. There was a statistically significant difference in BCVA over time $(P=0.01)$, a result of a two-letter loss at 1 month. The BCVA at 2 months PO was not statistically significantly different than the preoperative value (Tukey's Honestly Significant Difference post hoc test; $P>0.05)$. No eye had more than a two-letter loss of BCVA at 2 months. UCVA was statistically significantly better at both 1-month and 2-month PO stage relative to preoperative stage $(P<0.01)$, with an average of two lines of improvement at 2 months. In $79 \%$ of eyes (22/28), UCVA increased by more than one line, while no eye lost UCVA at 2 months.

The change in refraction is shown in Figure 4. There was no statistically significant difference in the spherical

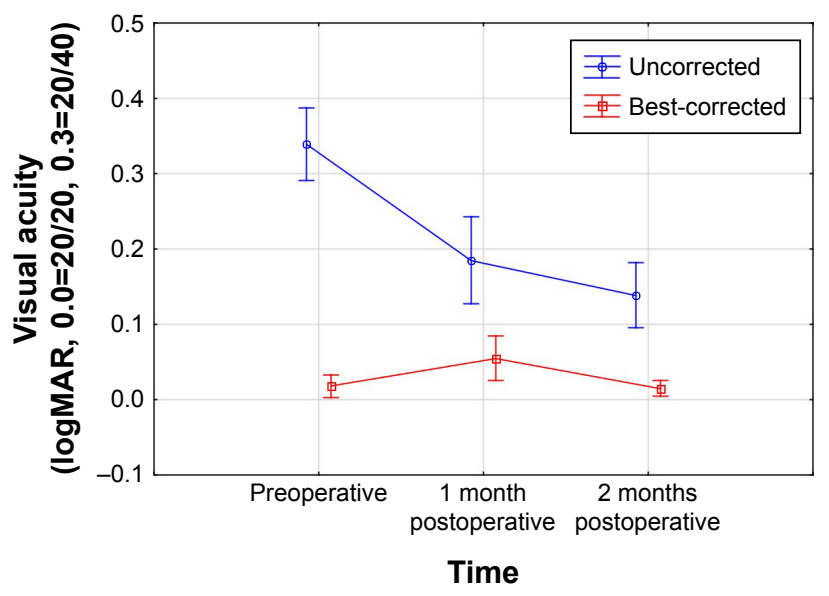

Figure 3 Visual acuity over time.

Note: Vertical bars denote $95 \%$ confidence intervals.

Abbreviation: logMAR, logarithm of the minimum angle of resolution.

equivalent refraction between the preoperative, 1-month and 2 -month visits $(P=0.34)$. The manifest refractive cylinder was statistically significantly lower than the preoperative condition at both the 1-month and 2-month visits $(P<0.01)$. The mean change in refractive cylinder from preoperative stage to the 2 -month PO value was 1.0 D. In $71 \%$ of eyes (20/28), the residual refractive cylinder at 2 months was $\leq 0.50 \mathrm{D}$, while in $93 \%$ of eyes $(26 / 28)$, it was $\leq 0.75$ D. Only one eye $(1.25 \mathrm{D})$ had a residual refractive cylinder $>1.00 \mathrm{D}$ at 2 months; the preoperative refractive cylinder in that eye was $2.00 \mathrm{D}$. The magnitude of the mean refractive cylinder was $0.25 \mathrm{D}$ lower at 2 months than at 1 month, which was statistically significant $(P=0.02)$, but perhaps not clinically significant, given the challenges in test-retest reliability of refractions. In $79 \%$ of eyes (22/28), the refractive cylinder

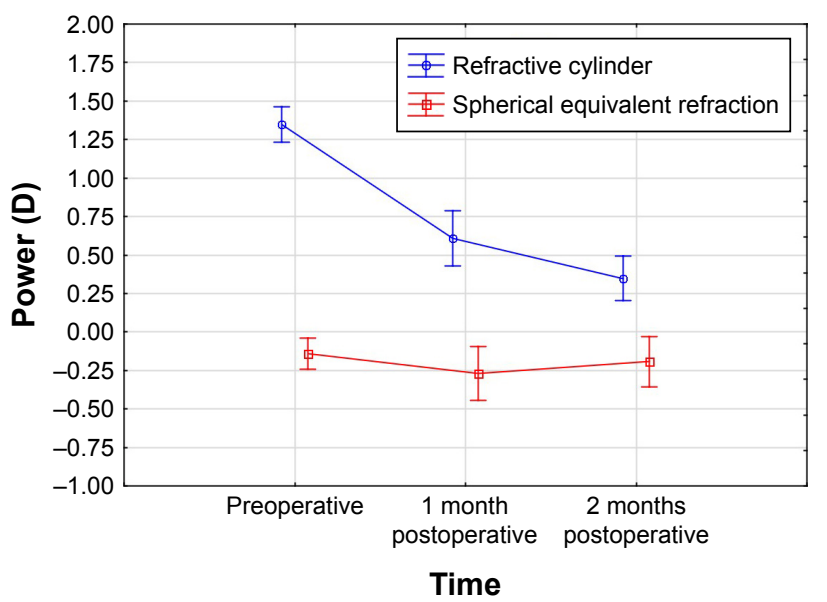

Figure 4 Spherical equivalent refraction and refractive cylinder over time. Note: Vertical bars denote $95 \%$ confidence intervals.

Abbreviation: D, diopters. 
magnitude at 2 months was equal to or lower than the magnitude at 1 month, and in only one eye was it $>0.50 \mathrm{D}$ higher $(0.75 \mathrm{D})$.

Preoperatively, $72 \%$ of subjects $(13 / 18)$ reported using their glasses for distance vision all of the time, while another $17 \%(3 / 18)$ reported using them most of the time. At the 2-month PO visit, two-thirds of the subjects (12/18) reported that they did not use glasses for distance vision. Only two subjects $(11 \%)$ reported using their glasses for distance vision all of the time. Half of the subjects $(9 / 18)$ reported that their spectacle use for distance vision at 2 months was "much lower" than their preoperative use, while two-thirds (12/18) reported it was "lower" or "much lower". Only one subject reported that spectacle use for distance vision at 2 months was "higher" than the preoperative use, but this result was ambiguous; the subject reported not using spectacles at all for distance vision at either the 1-month or 2-month visits, as well as reporting that use was "lower" than the preoperative use at the 1-month visit.

The vector change in keratometry from the preoperative stage to the 2-month PO visit was calculated for each of the three measurement devices. There was no statistically significant difference in the magnitude of the change in corneal astigmatism measured by device $(P=0.95)$ or the orientation of the change $(P=0.78)$. Given this result, it was sufficient to measure the refractive change relative to any one of the keratometric measures. The IOL Master keratometry was used for this purpose.

To determine how the keratometry changes were related to the refractive changes, two vectors were calculated. 1) The vector representing the change in refractive cylinder from the preoperative to 2 months postoperatively. 2) The vector representing the change in IOL Master keratometry in the same time period. These vectors were divided into orthogonal components, using double-angle vector math. The first component was along the steep refractive meridian which represents the intended effects of the corneal changes made using the arcuate incision. The second component was along the orthogonal meridian which represents the unintended effects of the corneal changes made using the arcuate incision - effectively an error. These data, and the percentage of the keratometry change relative to the refractive change in the meridian of the existing refractive cylinder, are shown in Table 2. Also shown is the refractive change relative to the steep refractive meridian.

The mean percentage change in refraction relative to the change in keratometry was $100.8 \%$, demonstrating how the arcuate corneal incision changed the refractive astigmatism
Table 2 Vector analysis of refractive and keratometric changes

\begin{tabular}{|c|c|c|c|c|}
\hline Properties & Mean & Minimum & Maximum & SD \\
\hline \multicolumn{5}{|l|}{ Change in refractive cylinder, $D$} \\
\hline Total & 1.21 & 0.29 & 2.00 & 0.42 \\
\hline $\begin{array}{l}\text { Along original refractive } \\
\text { cylinder meridian }\end{array}$ & 1.20 & 0.28 & 2.00 & 0.42 \\
\hline Orthogonal & -0.04 & -0.50 & 0.26 & 0.18 \\
\hline \multicolumn{5}{|c|}{ Change in corneal (keratometric) astigmatism, D } \\
\hline Total & 1.20 & 0.13 & 2.92 & 0.74 \\
\hline $\begin{array}{l}\text { Along original refractive } \\
\text { cylinder meridian }\end{array}$ & 1.16 & 0.09 & 2.92 & 0.77 \\
\hline Orthogonal & -0.07 & -0.55 & 0.38 & 0.22 \\
\hline $\begin{array}{l}\text { Percentage of keratometric } \\
\text { to refractive correction }\end{array}$ & 100.8 & 5.0 & 185.7 & 51.6 \\
\hline $\begin{array}{l}\text { Percentage of refractive } \\
\text { correction to original } \\
\text { refractive cylinder meridian }\end{array}$ & 88.0 & 28.0 & 137.0 & 23.8 \\
\hline
\end{tabular}

Note: ${ }^{\text {a }}$ The percentage of keratometric to refractive correction $=$ the change in corneal (keratometric) astigmatism along original refractive cylinder meridian/the change in refractive cylinder along original refractive cylinder meridian. Abbreviation: SD, standard deviation.

almost precisely as one would expect. This suggests that, in general, the keratometric change is predictive of the refractive change, so there was no apparent bias related to over- or undercorrection of corneal astigmatism versus refractive astigmatism. A single-sample $t$-test showed that the percentage change was not statistically significantly different from $100 \%$ $(P=0.94)$. Using the same test, the orthogonal (error) term was not statistically significantly different from zero for either the change in refractive cylinder $(P=0.28)$ or the change in keratometric astigmatism $(P=0.12)$. However, the change in the refractive astigmatism from the preoperative to the 2-month postop reading averaged $88 \%$; this was statistically significantly different from $100 \%(P=0.01)$. This indicates that the astigmatism was undercorrected by $\sim 10 \%$, suggesting that the nomogram might benefit from a $10 \%$ increase in treatment.

Figure 5 shows a plot of the magnitude of the vector change in IOL Master keratometric astigmatism, in terms of the orientation of the refractive change, versus the change in refractive cylinder at the same orientation, from the preop visit to the 2-month postop visit. As can be seen, the best fit is quite close to $1: 1$ (a slope of 0.95 ), but there is significant variability in the results between eyes.

There was one non-SAE. One subject returned 4 days after surgery with a history of redness and pain. A course of fortified vancomycin and tobramycin every 2 hours was initiated; the patient was seen daily for two days and then referred to a corneal specialist for further evaluation and treatment. The patient was diagnosed with a corneal ulcer over one of the incisions. At the 2-month visit, all symptoms had resolved, with a small corneal scar remaining over one 


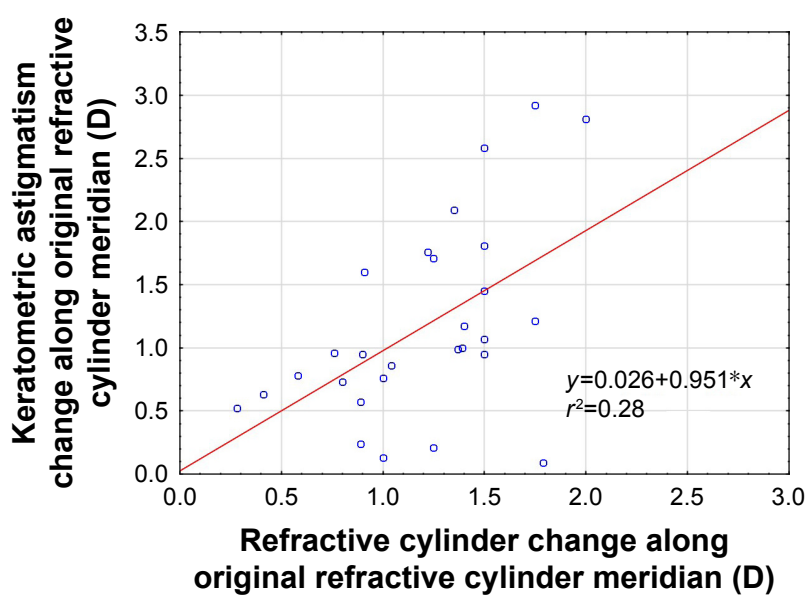

Figure 5 Change in keratometric astigmatism relative to change in refractive cylinder from the preoperative visit to the 2-month postoperative visit.

incision. There was a one-letter loss of BCVA (20/20 or 0.0 to $20 / 20-1$ or 0.02 ), while uncorrected acuity improved by one line, from $20 / 40(0.32)$ to $20 / 30(0.22)$ at 2 months. The subject reported no need for spectacles for distance vision at 1 month and 2 months postoperatively.

\section{Discussion}

The agreement between the three different keratometry measurements seen here appears consistent with the literature; the small difference between the Verion and the IOLMaster noted here has been previously documented. ${ }^{15}$ Another study comparing Verion, IOLMaster and autokeratometry found more differences between all three measurement devices than noted in the current study, though this was primarily a function of the autokeratometer. ${ }^{16}$ Using the IOL Master or the Verion unit for measuring corneal astigmatism appears equally effective.

The current study examined visual outcomes from laser arcuate incisions performed on patients who had previous cataract surgery. As the eyes were already pseudophakic, with stable refractions, the incisions were based on refractive cylinder rather than keratometric astigmatism. The primary outcome measure indicated that $80 \%$ of eyes gained a line or more of uncorrected visual acuity after the procedure, consistent with the observed reduction in refractive cylinder. UCVA improved on average by two lines at 2 months, which appears consistent with previous findings related to clinical outcomes with laser arcuate incisions. ${ }^{14}$ The mean UCVA of $0.14 \log$ MAR at 2 months was similar to that observed in the same time period with toric IOLs. ${ }^{8,17}$

With $70 \%$ of eyes having $\leq 0.5 \mathrm{D}$ of residual refractive cylinder after surgery, results appear as good as those reported in a meta-analysis of toric IOL outcomes, where a pooled average of $71 \%$ was calculated. ${ }^{18}$ However, the level of astigmatism correction in the current study was more limited (1.0-2.0 D) than for some of the toric studies included in that meta-analysis.

The mean change in both refractive and corneal astigmatism reported in the current study was $1.2 \mathrm{D}$, somewhat higher than the results previously reported. ${ }^{13}$ This could be attributed to the fact that refractive cylinder rather than corneal astigmatism was used for incision planning. In addition, the mean change in refractive cylinder was equal to the mean corneal astigmatism change in this study. That is not usually the case when the incision planning is based only on reducing corneal astigmatism. ${ }^{19}$ Finally, the correlation coefficient in the current study $(0.27)$ was higher than that observed in arcuate incision studies in the past $\left(0.17^{12}\right.$ and $\left.0.20^{20}\right)$. Some of this difference may be due to the use of a laser-specific nomogram (the Woodcock nomogram), but it may also be due to the alignment of the corneal incision with the axis of the residual refractive cylinder in the current study. With regard to the nomogram used, results suggest that a slightly higher value be used to achieve a more "centered" distribution of results, as the mean refractive reduction in the current study was $88 \%$.

At the 2-month follow-up, two-thirds of subjects (12/18) reported being spectacle independent, with the same percent reporting a decrease in their use of corrective lenses. This appears consistent with the spectacle independence rates for distance vision with toric IOLs. ${ }^{17}$

Figure 5 illustrates that while changes in mean refractive and keratometric cylinders are correlated, there is a fair bit of variability from eye to eye. Some of this variability is likely due to test-retest reliability in the measurement of corneal astigmatism and refractive cylinder. Some of this variability is also likely a function of changes in the posterior cornea, which may not be evident in the keratometry measures but which might affect the refraction. Posterior corneal astigmatism has been demonstrated to be a significant contributor to total corneal astigmatism. ${ }^{21}$ The effect of any incision is also dependent on patient-specific factors, particularly specific corneal properties that might affect wound healing. ${ }^{3}$ As such, while the nomogram was relatively good for balancing the mean correction, it would appear that some additional variables to help customize the nomogram to individual eyes would be helpful. ${ }^{20}$

There are limitations to the current study. The number of subjects was relatively low, sufficient to demonstrate a change in UCVA but insufficient to allow detailed post hoc analysis of the factors that might have influenced the effectiveness of the incisions; as noted herein, such an analysis might lead to better "customization" of incisions. Another 
limitation was that at the time of study design, it was felt that the 1-month and 2-month follow-up times would be sufficient to demonstrate refractive stability. Results suggest that stability might still be an issue in some eyes. A larger study with longer follow-up (eg, 3 months and 6 months) would be helpful to address these limitations.

\section{Conclusion}

Laser arcuate incisions in patients who have had previous cataract surgery appear to be an effective means of reducing refractive astigmatism, improving uncorrected visual acuity and reducing dependence on spectacles for distance vision for a high percentage of patients. Basing the arcuate incisions on residual refractive cylinder rather than corneal astigmatism appears to have some benefit.

\section{Acknowledgments}

Sarah Makari, OD, of Science in Vision, assisted in the preparation of the manuscript. Dr Clayton Blehm received an investigator-initiated study grant from Alcon to conduct this research (IIT proposal number 17077577). He provided funding to Science in Vision (Dr Potvin) to assist with study management, data analysis and preparation of this manuscript.

\section{Disclosure}

The authors report no conflicts of interest in this work.

\section{References}

1. Hoffmann PC, Hütz WW. Analysis of biometry and prevalence data for corneal astigmatism in 23,239 eyes. J Cataract Refract Surg. 2010;36(9): 1479-1485.

2. Atchison DA, Mathur A. Visual acuity with astigmatic blur. Optom Vis Sci. 2011;88(7):E798-E805.

3. Atchison DA, Guo H, Charman WN, Fisher SW. Blur limits for defocus, astigmatism and trefoil. Vision Res. 2009;49(19):2393-2403.

4. Hayashi K, Manabe S, Yoshida M, Hayashi H. Effect of astigmatism on visual acuity in eyes with a diffractive multifocal intraocular lens. J Cataract Refract Surg. 2010;36(8):1323-1329.

5. Woodward MA, Randleman JB, Stulting RD. Dissatisfaction after multifocal intraocular lens implantation. J Cataract Refract Surg. 2009; 35(6):992-997.

6. Kessel L, Andresen J, Tendal B, Erngaard D, Flesner P, Hjortdal J. Toric intraocular lenses in the correction of astigmatism during cataract surgery: a systematic review and meta-analysis. Ophthalmology. 2016;123(2):275-286

Clinical Ophthalmology

\section{Publish your work in this journal}

Clinical Ophthalmology is an international, peer-reviewed journal covering all subspecialties within ophthalmology. Key topics include: Optometry; Visual science; Pharmacology and drug therapy in eye diseases; Basic Sciences; Primary and Secondary eye care; Patient Safety and Quality of Care Improvements. This journal is indexed on
7. Maedel S, Hirnschall N, Chen YA, Findl O. Rotational performance and corneal astigmatism correction during cataract surgery: aspheric toric intraocular lens versus aspheric nontoric intraocular lens with opposite clear corneal incision. J Cataract Refract Surg. 2014;40(8):1355-1362.

8. Mingo-Botín D, Muñoz-Negrete FJ, Won Kim HR, Morcillo-Laiz R, Rebolleda G, Oblanca N. Comparison of toric intraocular lenses and peripheral corneal relaxing incisions to treat astigmatism during cataract surgery. J Cataract Refract Surg. 2010;36(10):1700-1708.

9. Grewal DS, Basti S. Comparison of morphologic features of clear corneal incisions created with a femtosecond laser or a keratome. $J$ Cataract Refract Surg. 2014;40(4):521-530.

10. Mastropasqua L, Toto L, Mastropasqua A, et al. Femtosecond laser versus manual clear corneal incision in cataract surgery. J Refract Surg. 2014;30(1):27-33.

11. Nagy ZZ, Filkorn T, Takács AI, et al. Anterior segment OCT imaging after femtosecond laser cataract surgery. J Refract Surg. 2013; 29(2):110-112.

12. Chan TC, Cheng GP, Wang Z, Tham CC, Woo VC, Jhanji V. Vector analysis of corneal astigmatism after combined femtosecond-assisted phacoemulsification and arcuate keratotomy. Am J Ophthalmol. 2015;160(2):250.e-255.e.

13. Rückl T, Dexl AK, Bachernegg A, et al. Femtosecond laser-assisted intrastromal arcuate keratotomy to reduce corneal astigmatism. J Cataract Refract Surg. 2013;39(4):528-538.

14. Yoo A, Yun S, Kim JY, Kim MJ, Tchah H. Femtosecond laser-assisted arcuate keratotomy versus toric IOL implantation for correcting astigmatism. J Refract Surg. 2015;31(9):574-578.

15. Mueller A, Thomas BC, Auffarth GU, Holzer MP. Comparison of a new image-guided system versus partial coherence interferometry, Scheimpflug imaging, and optical low-coherence reflectometry devices: keratometry and repeatability. J Cataract Refract Surg. 2016;42(5):672-678.

16. Asena L, Güngör SG, Akman A. Comparison of keratometric measurements obtained by the Verion Image Guided System with optical biometry and auto-keratorefractometer. Int Ophthalmol. Epub 2016 Jun 7.

17. Ahmed II, Rocha G, Slomovic AR, et al. Visual function and patient experience after bilateral implantation of toric intraocular lenses. $J$ Cataract Refract Surg. 2010;36(4):609-616.

18. Visser N, Bauer NJ, Nuijts RM. Toric intraocular lenses: historical overview, patient selection, IOL calculation, surgical techniques, clinical outcomes, and complications. J Cataract Refract Surg. 2013;39(4): 624-637.

19. Leon P, Pastore MR, Zanei A, et al. Correction of low corneal astigmatism in cataract surgery. Int J Ophthalmol. 2015;8(4):719-724.

20. Day AC, Stevens JD. Predictors of femtosecond laser intrastromal astigmatic keratotomy efficacy for astigmatism management in cataract surgery. J Cataract Refract Surg. 2016;42(2):251-257.

21. Zheng T, Chen Z, Lu Y. Influence factors of estimation errors for total corneal astigmatism using keratometric astigmatism in patients before cataract surgery. J Cataract Refract Surg. 2016;42(1):84-94. 